\title{
Pseudognaphalium fastigiatum (Compositae: GnaphalieAe) es EL NOMBRE CORRECTO PARA $P$. CABRERAE
}

\author{
NÉSTOR D. BAYÓN¹ y DANIEL A. GIULIANO1
}

\begin{abstract}
Summary: Pseudognaphalium fastigiatum (Compositae: Gnaphalieae) is the correct name for $P$. cabrerae. Pseudognaphalium cabrerae (S. E. Freire) Deble was proposed as a new combination in 2006 for the South American Gnaphalium fastigiatum Philippi (1895), non Thunb. (1800), nec Schrank (1824), also called G. philippii Cabrera (1941), non Gand. (1918). However, P. cabrerae is invalid under ICBN rules. Pseudognaphalium fastigiatum is proposed as the correct name for this taxon.
\end{abstract}

Key words: Compositae, Gnaphalium, Pseudognaphalium, nomenclature.

\begin{abstract}
Resumen: La nueva combinación Pseudognaphalium cabrerae (S.E. Freire) Deble fue propuesta en 2006 para denominar a la especie sudamericana Gnaphalium fastigiatum Philippi (1895), non Thunb. (1800), nec Schrank (1824), también llamada G. philippii Cabrera (1941), non Gand. (1918). Sin embargo, el nombre $P$. cabrerae es inválido bajo las reglas del CINB, siendo Pseudognaphalium fastigiatum indicado como el nombre correcto para este taxon.
\end{abstract}

Palabras clave: Compositae, Gnaphalium, Pseudognaphalium, nomenclatura.

Pseudognaphalium (Compositae: Gnaphalieae) fue propuesto por Kirpicznikov (Kirpicznikov \& Kuprijanova, 1950) como un nuevo género al que Anderberg (1991) transfirió numerosas especies, las que generalmente fueron tratadas dentro del género Gnaphalium L. (Cabrera, 1963, 1971, 1974, 1978; Dillon \& Sagástegui-Alva, 1991; Freire, 1995, 1998; Freire et al., 2005, 2012; Bayón, 2009). Como parte del tratamiento del género Pseudognaphalium para la Flora de San Juan se halla Pseudognaphalium cabrerae (S.E. Freire) Deble (2006), especie de Argentina y Chile que fue descripta originalmente por Philippi en 1895 en forma ilegítima bajo el nombre de Gnaphalium fastigiatum. Ese nombre ya había sido utilizado en forma previa por Thunberg en 1800 y por Schrank en 1824. Posteriormente, Cabrera (1941) le asigna el nuevo nombre de Gnaphalium philippii, sin tener en cuenta que se trataba de otro homónimo, dado que Gandoger (1918) había ocupado previamente

\footnotetext{
1 Área de Botánica, Departamento de Ciencias Biológicas, Facultad de Ciencias Agrarias y Forestales, Universidad Nacional de La Plata, Avda. 60 y 119, 1900 La Plata. nbayon@isis.unlp.edu.ar
}

dicho epíteto para una especie europea. Freire (1999) propone de manera inválida (McNeill et al., 2006, Art. 33.4) el nuevo nombre de Gnaphalium cabrerae; sin embargo, éste había sido válidamente publicado en la Errata et Corrigenda de la Flora Fanerogámica Argentina (Freire, 1995). Más tarde, Deble (2006) transfiere la especie al género Pseudognaphalium, bajo el nombre de $P$. cabrerae, basándose en la publicación inválida del nombre (Freire, 1999); esta combinación está inválidamente publicada según el Art. 33.7 (a) (McNeill et al., 2006). Asimismo, este autor (Deble, 2006) no tuvo en cuenta lo establecido por el Artículo 53.6, Nota 3 (McNeill et al., 2006), que sostiene que un homónimo renombrado o rechazado de acuerdo con este Artículo continúa siendo legítimo y adquiere precedencia sobre un sinónimo posterior del mismo rango, si se lo transfiere a otro género o especie. Por todo lo expuesto el nombre correcto de esta especie debe ser Pseudognaphalium fastigiatum, constituyendo P. cabrerae un nombre inválido.

Pseudognaphalium fastigiatum N. Bayón, nom. nov. $\equiv$ Gnaphalium fastigiatum Phil., Anales 
Univ. Chile 90: 15. 1895, hom. illeg., non Thunb. (1800), nec Schrank (1824) 三 Gnaphalium philippii Cabrera, Revista Mus. La Plata, Secc. Bot. 4: 164. 1941, hom. illeg. $\equiv$ Gnaphalium cabrerae S.E. Freire, Flora Fanerogámica Argentina 14, Errata et Corrigenda 4. 1995 三 Pseudognaphalium cabrerae (S.E. Freire) Deble, Balduinia 6: 29. 2006. nom. inval. Tipo: Chile. Santiago, Quinta Normal, III-1878, Philippi s.n. (Holotypus, SGO 64449!).

Esta especie nativa de Argentina y Chile (Cabrera, 1963; Freire, 1995; Cerana \& Ariza, Espinar, 2008) se caracteriza por tener hojas angostamente elípticas, agudas, con escasos pelos glandulares ocultos debajo de la lana, y capítulos en glomérulos reunidos en laxas cimas corimbiformes terminales, con involucro de 3-4 $\mathrm{mm}$ alt. $\times$ 3-4 mm diám. Es una especie cercana a Pseudognaphalium leucopeplum (Cabrera) Anderb., de la que se diferencia porque esta última tiene el involucro de mayor tamaño, de 5-5,5 $\mathrm{mm}$ alt. $\times 6 \mathrm{~mm}$ diám.

\section{Agradecimientos}

A Luis Ariza Espinar, pues a raíz de su inquietud surge la necesidad de realizar esta comunicación, como así también a Susana Freire por la lectura crítica del manuscrito. El proyecto en el que se enmarca esta nota ha sido financiado por la Universidad Nacional de La Plata (Programa de Incentivos, Secretaría de Políticas Universitarias, Ministerio de Educación, Argentina).

\section{Bibliografía}

ANDERBERG, A. A. 1991. Taxonomy and phylogeny of the tribe Gnaphalieae (Asteraceae). Opera Bot. 104: 146-148.

BAYÓN, N. D. 2009. Compositae: Gnaphalium. En: FREIRE, S.E. \& A.M. MOLINA (eds.), Flora Chaqueña 23 (2): 559-561. Colecc. Ci. Inst. Nac. Tecnol. Agropecu., Buenos Aires.

CABRERA, A.L. 1963. Compositae: Gnaphalium. En: CABRERA, A.L. (ed.), Flora de la Provincia de Buenos 4 (6): 161-166. Colecc. Ci. Inst. Nac. Tecnol. Agropecu., Buenos Aires.

CABRERA, A.L. 1971. Compositae: Gnaphalium. En:
CORREA, M.N. (ed.), Flora Patagónica 8 (7): 109-117. Colecc. Ci. Inst. Nac. Tecnol. Agropecu., Buenos Aires.

CABRERA, A.L. 1974. Compositae: Gnaphalium. En: BURKART, A. (ed.), Flora Ilustrada de Entre Ríos 6 (6): 315-317. Colecc. Ci. Inst. Nac. Tecnol. Agropecu., Buenos Aires.

CABRERA, A.L. 1978. Compositae: Gnaphalium. En: CABRERA, A.L. (ed.), Flora de la Provincia de Jujuy 13 (10): 275-288. Colecc. Ci. Inst. Nac. Tecnol. Agropecu., Buenos Aires.

CERANA, M.M. \& L. ARIZA ESPINAR. 2008. Familia Asteraceae: Tribu Inuleae (Achyrocline, Gamochaeta y Gnaphalium). Pródr. Fl. Fanerogám. Argent. Central 4: 5-14, 26-52. Museo Botánico, Córdoba.

DEBLE, L. P. 2006. Un novo nome e duas novas combinações na tribu Gnaphalieae (Asteraceae). Balduinia 6: 29.

Dillon, M.O. \& A. SAGÁStEGUI-ALVA. 1991. Sinopsis de los géneros de Gnaphaliinae (Asteraceae-Inuleae) de Sudamérica. Arnaldoa 1: 5-91.

FREIRE, S.E. 1995. Asteraceae. Tribu IV. Inuleae. En: HUNZIKER, A. T. (ed.), Flora Fanerogámica Argentina 14, Errata et Corrigenda 4. Proflora, Córdoba.

FREIRE, S.E. 1998. Tribu Inuleae (Compositae). En: SPICHIGER, R. \& L. RAMELLA (eds.), Flora del Paraguay 27: 9-100. Conservatoire et Jardín Botaniques, Geneve, y Missouri Botanical Garden, St. Louis.

FREIRE, S.E. 1999. Asteraceae. Inuleae. En: ZULOAGA, F.O. \& O. MORRONE (eds.), Catálogo de las Plantas Vasculares de la República Argentina. 2. (Dicotyledoneae). Monogr. Syst. Bot. Missouri Bot. Gard. 74: 98-353.

FREIRE, S.E., G. SANCHO, E. URTUBEY, N.D. BAYÓN, L. KATINAS, D.A. GIULIANO, D.G. GUTIÉRREZ, A.A. SÁENZ, L. IHARLEGUI, C. MONTI \& G. DELUCCHI. 2005. Catalogue of Asteraceae of Chacoan Plain, Argentina. Compositae Newslett. 43: 1-126.

FREIRE, S.E., N.D. BAYÓN, C. MONTI, D.A. GIULIANO, L. ARIZA ESPINAR, A.A. SÁENZ, M.V. PEREA \& G. DELUCCHI. 2012. Sinopsis de las Asteráceas de la Provincia de Catamarca. Editorial Científica Universitaria de la Universidad Nacional de Catamarca, Catamarca.

KIRPICZNIKOV, M. E. \& L. A. KUPRIJANOVA. 1950. Morphological-geographical and palynological contributions to the understanding of the genera of the subtribe Gnaphaliinae (en ruso). Trudy Bot. Inst. Akad. Nauk S.S.S.R., Ser. 1, Fl. Sist. Vyssh. Rast. 9: 7-37. 
N. D. Bayón y D. A. Giuliano - Nombre correcto para Pseudognaphalium cabrerae

MCNEILL, J., F. R. BARRIE, H. M. BURDET, V. DEMOULIN, D. L. HAWKSWORTH, K. MARHOLD, D. H. NICOLSON, J. PRADO, P. C. SILVA, J. E. SKOG, J. H. WIERSEMA \& N. J. TURLAND (eds.). 2006. International Code of Botanical Nomenclature (Vienna Code) adopted by the Seventeenth International Botanical Congress
Vienna, Austria, July 2005. Regnum Vegetabile 146. Gantner Verlag, Ruggell, Liechtenstein.

Recibido el 18 de noviembre de 2012, aceptado el 8 de febrero de 2013. 
